Es war einmal ein Königssohn, der bekam Lust in der Welt umher zu ziehen

DIE DEUTSCHEN d-V2-SÄTZE: SYNCHRONE UND DIACHRONE ÜBERLEGUNGEN ${ }^{1}$

\begin{abstract}
In diesem Aufsatz geht es um Sätze, deren Vorfeld mit einem anaphorischen d-Pronomen des Typs der/die/das besetzt ist und die - im Gegensatz zu Relativsätzen - Zweitstellung des Finitums aufweisen (d-V2-Sätze), wie in: Ich habe einen Bekannten, der fährt einen Porsche. Sätze dieser Art werden in drei Perioden der Sprachgeschichte untersucht. Das Korpus besteht aus Texten aus dem Frühneuhochdeutschen, dem 19. Jahrhundert und der deutschen Gegenwartssprache. In allen drei Perioden kommen d-V2Sätze vor. Sie werden nach ausgewählten Kriterien untersucht und mit Relativsätzen verglichen. Es werden Bedingungen formuliert, unter denen Relativsätze durch d-V2-Sätze substituiert werden können.

The present article deals with clauses which have an anaphoric d-pronoun (der/die/das) in the Vorfeld (front field) and whose finite verb is - in contrast to relative clauses - in second position (d-V2-clauses), as in: Ich habe einen Bekannten, der fährt einen Porsche. Clauses of this type are analysed in three historical periods. The corpus consists of texts from Early New High German, from the 19th century and from present-day German. In all three periods, d-V2-clauses occur in the data. They are examined according to various syntactic criteria and compared to relative clauses. Finally, conditions are formulated that allow for the substitution of relative clauses by $\mathrm{d}$-V2-clauses.
\end{abstract}

\title{
1. Gegenstand der Untersuchung
}

Gegenstand meiner Untersuchung sind Sätze, die mit einem d-Pronomen (vgl. Wiltschko 1998, S. 144; Penner 1996, S. 23) des Typs der/die/das eingeleitet werden, die aber - im Gegensatz zu subordinierten Relativsätzen - die Zweitstellung des Finitums aufweisen (vgl. Beispiel (1)). Solche Konstruktionen werden hier als d-V2-Sätze bezeichnet:

Als sie ins Schloss gekommen waren, führte sie es hinauf zu drei Kammern, die lagen von unten bis oben voll vom schönsten Flachs. (KHM 14, S. 84)

Im ersten Teil meines Aufsatzes wird auf Gemeinsamkeiten zwischen d-V2-Sätzen und Relativsätzen eingegangen; anschließend untersuche ich Bedingungen, unter denen d-V2-Sätze anstelle von Relativsätzen verwendbar sind.

In der Fachliteratur gibt es unterschiedliche Meinungen darüber, wie man d-V2-Sätze behandeln soll. Lehmann (1995, S. 1204) und Gärtner (2000, S. 139) weisen auf Eigenschaften hin, die es erlauben, solche Strukturen zu den Relativsätzen zu zählen. Reis (1997) zeigt den zweideutigen Charakter der d-V2-Sätze auf. Einerseits haben sie hauptsatztypische Verbstellung, andererseits verhalten sie sich semantisch wie eingebettete Sätze. Zifonun (2001) und Schieb (1978) stufen sie als Hauptsätze ein, die durch ein anaphorisches Pronomen mit dem vorausgehenden Satz verbunden sind.

\footnotetext{
' Für hilfreiche Kommentare zu diesem Beitrag möchte ich mich bei Hardarik Blühdorn herzlich bedanken.
} 
Meine Untersuchung stützt sich nicht nur auf gegenwartssprachliche, sondern auch auf historische Daten. Mein Korpus besteht aus Texten aus drei Untersuchungsperioden: zwei Prosaromanen (Melusine und Fortunatus) aus der frühneuhochdeutschen Zeit, den Kinder- und Hausmärchen der Brüder Grimm (Nr. 1 bis Nr. 50) für das 19. Jahrhundert und Uwe Timms Roman Heißer Sommer (1974) für die heutige Zeit. Dazu wurde auch eine Sammlung von Interviews in Betracht gezogen, die von Bredel und Dittmar in den $90 \mathrm{er}$ Jahren aufgenommen und transkribiert wurden und mir freundlicherweise für meine Untersuchung zur Verfügung gestellt wurden. Beispiele aus dieser Sammlung von Interviews werden in meinem Aufsatz nach dem Transkriptionssystem angeführt, das Bredel und Dittmar für ihr Korpus verwendet haben. Ergänzend werden punktuell einige Beispiele aus dem Spiegel (Jahrgang 1993; IDS-Korpus) angeführt.

In den Grimmschen Märchen treten d-V2-Sätze viel häufiger auf als in den anderen Vergleichstexten meines Korpus. Das ist mit stilistischen Absichten der Autoren zu erklären. Die Brüder Grimm haben die Texte mehrmals bearbeitet, um den Stil volkstümlicher zu gestalten (vgl. Wilhelm Grimms Aussage in der Einleitung der 1837 veröffentlichten dritten Ausgabe: ,Außerdem sind viele der frühern Stücke abermals umgearbeitet und durch Zusätze und einzelne, aus mündlichen Erzählungen gewonnene Züge ergänzt und bereichert", KHM 1989, S. 24). Zu den stilistischen Mitteln, die bei der Bearbeitung hinzugefügt wurden, gehören auch d-V2-Sätze, die anstelle von subordinierten Relativsätzen von Ausgabe zu Ausgabe häufiger werden (vgl. Ravetto 2006, S. 80-84).

Die diachrone Analyse zeigt, dass d-V2-Sätze in allen Perioden des Untersuchungszeitraums auftreten und somit eine auffällige Kontinuität aufweisen. In jedem Text des Korpus wird die relative Häufigkeit von d-V2-Sätzen geprüft und mit derjenigen von Relativsätzen verglichen.

Die folgende Tabelle zeigt das Vorkommen von d-V2-Sätzen und Relativsätzen in meinem Korpus für die drei betrachteten Perioden:

(2) d-V2-Sätze und Relativsätze im untersuchten Korpus

\begin{tabular}{|l|l|l|l|l|}
\hline & $\begin{array}{l}\text { Frühneu- } \\
\text { hochdeutsch }\end{array}$ & $\begin{array}{l}\text { 19. Jahr- } \\
\text { hundert }\end{array}$ & $\begin{array}{l}\text { Gegenwarts- } \\
\text { sprache }\end{array}$ & Summe \\
\hline d-V2-Sätze & 280 & 84 & 116 & 480 \\
\hline Relativsätze & 418 & 172 & 263 & 853 \\
\hline
\end{tabular}

Die Pronominalformen der/die/das, die d-V2-Sätze einleiten, sind mit den Relativpronomen gleichlautend, wie Tabelle (3) zeigt:

(3)

\begin{tabular}{|l|l|l|l|}
\hline \multicolumn{2}{|l|}{} & \multicolumn{2}{|l|}{ Relativpronomen } \\
\cline { 2 - 4 } \multicolumn{2}{|l|}{} & + & - \\
\hline Demonstrativpronomen & + & der, die, das .... & dieser, diese, dieses .... \\
\cline { 2 - 4 } & - & welcher, welche, welches; was, wo... & $\ldots$ \\
\hline
\end{tabular}

(aus: Gärtner 2000, S. 131) 
Nach Gärtner können die Pronominalformen der/die/das als Demonstrativa und als Relativpronomen auftreten. Dank ihres doppelten Gebrauchs bilden sie ein interessantes Forschungsfeld in synchroner wie in diachroner Sicht.

Im vorliegenden Aufsatz weise ich auf relevante quantitative Unterschiede zwischen d-V2-Sätzen und Relativsätzen hin und untersuche, mit welchen syntaktischen bzw. semantischen Faktoren das Auftreten der Zweitstellung des finiten Verbs nach dPronomen verbunden ist. Es soll ein Beitrag dazu geleistet werden, den Status von d-V2Sätzen durchsichtiger zu machen.

\section{2. Ähnlichkeiten von d-V2-Sätzen und Relativsätzen}

Zwischen d-V2-Sätzen und Relativsätzen besteht eine enge Verwandtschaft. In d-V2Sätzen besetzt das d-Pronomen das Vorfeld, wie Beispiel (4) zeigt (VF - Vorfeld, LK linke Satzklammer, MF - Mittelfeld, RK - rechte Satzklammer):

Da fielen noch mehr Männer herab, $[\mathbf{d i e}]_{\mathrm{VF}}[\text { hatten }]_{\mathrm{LK}}[$ neun Totenbeine und zwei Totenköpfe $]_{\mathrm{MF}}[\emptyset]_{\mathrm{RK}}$, setzten auf, und spielten Kegel. (KHM 4, S. 40)

Nach der überzeugenden Analyse von Wöllstein-Leisten et al. (1997, S. 54) stehen auch Relativpronomina im Vorfeld. Die linke Satzklammer von Relativsätzen ist dieser Analyse zufolge unbesetzt:

Aber der arme Königssohn fand oben nicht seine liebste Rapunzel, sondern die Zauberin, $[\text { die }]_{\mathrm{VF}}[\emptyset]_{\mathrm{LK}}[\text { ihn mit bösen und giftigen Blicken }]_{\mathrm{MF}}[\text { ansah }]_{\mathrm{RK}}$. (KHM 12, S. 78)

Die Pronominalformen der/die/das können in Relativsätzen wie auch in d-V2-Sätzen von Präpositionen regiert sein, wie etwa in den Beispielen (6) und (7):

(6) Rechts zog sich eine Bücherwand in einem kleineren Raum, in dem ein großer Eßtisch stand. (Heißer Sommer, S. 168)

(7) Hinter ihm geht sein Assistent, hinter dem geht seine wissenschaftliche Hilfskraft. (HeiBer Sommer, S. 32)

In d-V2-Sätzen wie in Relativsätzen knüpft das d-Pronomen anaphorisch an eine Konstituente des vorangehenden Satzes an. Seine morphologische Form wird teilweise vom Antezedens und teilweise durch seine Rolle im Nachfolgersatz bestimmt (vgl. Zifonun in diesem Band; Lehmann 1995, S. 1204; Zifonun 2001, S. 27 f.). Mit dem wiederaufgenommenen Bezugselement stimmt es in Genus und Numerus überein. Für Wiltschko (1998) ist die Kongruenzfähigkeit eine der Haupteigenschaften der Relativpronomen. Als Beispiel führt sie das Holländische an, das über zwei Demonstrativformen verfügt, eine schwache (de/het), die nur als Demonstrativum auftreten kann, und eine flektierte (die/dat), die mit ihrem Bezugsausdruck kongruiert und als Relativpronomen verwendbar ist. Seinen Kasus erhält das d-Pronomen sowohl im Relativsatz als auch im d-V2Satz vom Verb oder von einer Präposition in dem von ihm eingeleiteten Satz: 


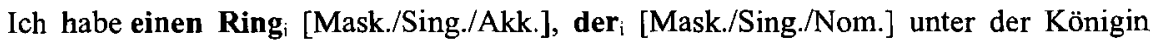
Fenster lag, in der Hast mit hinunter geschluckt. (KHM 17, S. 97)

(9) Ich habe eine entfernte Bekannte ${ }_{i}\left[\mathrm{Fem} . /\right.$ Sing./Akk.], die ${ }_{i}[\mathrm{Fem} . /$ Sing./Nom.] hat Schwierigkeiten. (Heißer Sommer, S. 60)

Das Antezedens kann dem wiederaufnehmenden Pronomen unmittelbar vorangehen (vgl. Beispiele (10) und (11)); es ist aber auch Distanzstellung des Pronomens zum Antezedens möglich, wie Beispiele (12) und (13) zeigen. Dies ist eine weitere Eigenschaft, die d-V2-Sätze mit Relativsätzen teilen.

(10) Fracht ich also ein türkn + nach einer straße in der/ in berlin in der stadt in der ich jeborn war. (Bredel/Dittmar: B24O LORE, S. 50)

(11) Ich kannte einen jungen Mann, dem hat's der Türspalt angetan. (Heißer Sommer, S. 264)

(12) Und als er das Bildnis der Jungfrau erblickte, das so herrlich war und von Gold glänzte, da fiel er alsbald ohnmächtig auf die Erde nieder. (KHM 6, S. 47)

(13) Wie soll ich übers Herz bringen, meine eigenen lieben Kinder den wilden Tieren im Wald zu überliefern, die würden sie bald zerrissen haben. (KHM 15, S. 87)

d-V2-Sätze können sich ebenso wie Relativsätze auf den ganzen vorangehenden Satz oder auf umfangreiche Teile davon beziehen und können in diesem Sinne weiterführend sein (vgl. Holler 2005 und in diesem Band; Bergmann 1985). In Relativsätzen werden hierfür gegenwartssprachlich allerdings ausschließlich w-Relativpronomina (was) bzw. Relativadverbien (womit, wobei usw.) verwendet (Beispiel (14)). D-Pronomina knüpfen im Gegenwartsdeutschen nur in V2-Sätzen an satzförmige Antezedentien an (Beispiel (15)):

(14) So diente sich der Abgeordnete aus dem Verteidigungsausschuß 1980 diversen Waffenfirmen als Interessenvertreter an, was aus der Sicht der verblüfften Kriegsgerätehersteller eindeutig mit dem Ansinnen um finanzielle Stïtzen verbunden war. (Der Spiegel, 1993, Heft 1, S. 18)

(15) Die Polizei ist auf den sogenannten Großen Lauschangriff bei der vorbeugenden Aufklärung von Verbrechen offenbar nicht angewiesen. Das fand der rheinland-pfälzische FDP-Justizminister Peter Caesar bei einer Umfrage im Landeskriminalamt und bei Gerichten heraus. (Der Spiegel, 1993, Heft 46, S. 16)

In den frühneuhochdeutschen Texten (Beispiel (16)) und - seltener - in den Grimmschen Märchen (Beispiel (17)) kommt hingegen das Pronomen das für die Wiederaufnahme des vorangehenden Satzes vor:

(16) du soelt mein hie warten piß ich herwiderumb komm / das auch als ich hoff gar in kürcz geschehen soel. (Melusine, S. 107)

(Du sollst auf mich hier warten, bis ich zurückgekommen bin, was hoffentlich in Kürze geschehen soll.)

(17) Da steht ihr Haus, unten sind die Nußhecken, das wirst du ja wissen. (KHM 26, S. 134) 
Auch in semantischer Hinsicht zeigt sich die Ahnlichkeit von a-v L-Sarzen mu Ncrativsätzen. Wie diese können sie sich in Bezug auf ihr Antezedens restriktiv (Beispiele (18) und (19)) oder appositiv (Beispiele (20) und (21)) verhalten.

(18) Der Mann, der Ullrich gegenübersaß, hatte inzwischen den Fahrplan beiseite gelegt. (Heißer Sommer, S. 303)

(19) Sie kenne jemanden, der suche Leute für eine Wohngemeinschaft. (Heißer Sommer, S. 228)

(20) Als es aber Mitternacht war und alles schlief, da sah die Kinderfrau, die in der Kinderstube neben der Wiege saß und allein noch wachte, wie die Türe aufging. (KHM 11, S. 73)

(21) Allerdings habe sie Glück gehabt und die Haut von ihrer Mutter geerbt, die wird ganz schnell braun. (Heißer Sommer, S. 88)

Trotz aller Gemeinsamkeiten werden d-V2-Sätze und Relativsätze aber recht unterschiedlich verwendet, wie im Folgenden anhand von Korpusdaten gezeigt wird.

\section{3. d-V2-Sätze und Relativsätze in den Korpusdaten}

In diesem Abschnitt werden d-V2-Sätze und Relativsätze in den Texten des Korpus nach den folgenden Kriterien ${ }^{2}$ untersucht und verglichen:

- Stellung in der Linearstruktur des Satzes

- Kontakt- oder Distanzstellung zum Antezedens

- Ausdrucksklasse des Antezedens

- Rektion der d-Pronomen durch eine Präposition

- Restriktiver bzw. appositiver Gebrauch des Satzes

Zunächst zur Stellung von d-V2-Sätzen und Relativsätzen in der Linearstruktur des Gesamtsatzes. Während Relativsätze im Vorfeld und im Mittelfeld stehen können (Beispiel (22) und (23)), sind d-V2-Sätze auf die Position nach der rechten Satzklammer beschränkt (Beispiel (24)). Dies gilt gleichermaßen für die älteren und für die neueren Texte des Korpus, wie die tabellarische Übersicht zeigt:

(22) Wer sich mit dem einläßt, ist gnadenlos weg. (Der Spiegel, 1993, Heft 1, S. 18)

(23) Die Nutte, die in dem dunklen Eingang eines Fachwerkhauses stand, hatte der Gruppe nachgerufen. (Heißer Sommer, S. 99)

(24) In dem Schloße steckten große Schätze, von Geistern bewacht, die würden dann frei. (KHM 4, S. 38)

\footnotetext{
${ }^{2}$ Hier werden nur die wichtigsten Faktoren berücksichtigt. Für weitere Vergleichskriterien wie Kasus der d-Pronomen und Satzlänge vgl. Ravetto (2006).
} 
(25) Stellung von d-V2-Sätzen und Relativsätzen

\begin{tabular}{|l|l|l|l|l|}
\hline & $\begin{array}{l}\text { Frühneuhoch- } \\
\text { deutsch }\end{array}$ & $\begin{array}{l}\text { 19. Jahr- } \\
\text { hundert }\end{array}$ & $\begin{array}{l}\text { Gegenwarts- } \\
\text { sprache }\end{array}$ & Summe \\
\hline $\begin{array}{l}\text { d-V2 nach der rechten } \\
\text { Satzklammer }\end{array}$ & $279(99,7 \%)$ & $84(100 \%)$ & $116(100 \%)$ & $479(99,8 \%)$ \\
\hline $\begin{array}{l}\text { d-V2 im Vorfed oder im } \\
\text { Mittelfeld }\end{array}$ & $1(0,3 \%)$ & 0 & 0 & $1(0,2 \%)$ \\
\hline $\begin{array}{l}\text { RS nach der rechten Satz- } \\
\text { klammer }\end{array}$ & $382(91 \%)$ & $118(69 \%)$ & $200(76 \%)$ & $700(82 \%)$ \\
\hline $\begin{array}{l}\text { RS im Vorfed oder im Mit- } \\
\text { telfeld }\end{array}$ & $36(9 \%)$ & $54(31 \%)$ & $63(24 \%)$ & $153(18 \%)$ \\
\hline
\end{tabular}

Die Tabelle zeigt, dass d-V2-Sätze auf die Position nach der rechten Satzklammer festgelegt sind, während Relativsätze zu etwa einem Sechstel im Vor- und Mittelfeld auftreten.

Sowohl d-V2-Sätze als auch Relativsätze können in Distanzstellung zu ihrem Antezedens stehen. Die Distanzstellung ist in allen untersuchten Epochen belegt, wie in den folgenden Beispielsätzen und Tabellen gezeigt wird. Beispiele (26) und (27) zeigen die Distanzstellung eines d-V2-Satzes zu seinem Antezedens im Frühneuhochdeutschen und im 19. Jahrhundert. Beispiele (28) und (29) illustrieren die Distanzstellung eines Relativsatzes im Frühneuhochdeutschen und in der Gegenwartssprache:

(26) nvn sahe der künig den sperber dort steen auf einer stangen der was gar schoen vnd auch zemal groß. (Melusine, S. 158)

(Nun sah der König den Sperber dort auf einer Stange stehen, der war sehr schön und besonders groß.)

(27) Nun trug sich zu, daß ein König in dem Wald jagte, der hatte einen großen Windhund. (KHM 9, S. 65)

(28) vnd bracht den leib wider zu krefften / der doch swach vnd bloed was. (Fortunatus, S. 538)

(und brachte den Körper wieder zu Kräften, der schwach und kraftlos war.)

(29) Das offiziell verdrängte Elend muß sichtbar gemacht werden, das dieses Gesellschaftssystem permanent verbreitet. (Heißer Sommer, S. 111)

(30) Kontakt- und Distanzstellung der d-V2-Sätze und der Relativsätze zu ihrem Antezedens.

\begin{tabular}{|l|l|l|l|l|}
\hline & $\begin{array}{l}\text { Frühneu- } \\
\text { hochdeutsch }\end{array}$ & $\begin{array}{l}\mathbf{1 9 . J a h r -} \\
\text { hundert }\end{array}$ & $\begin{array}{l}\text { Gegenwarts- } \\
\text { sprache }\end{array}$ & Summe \\
\hline d-V2 in Kontaktstellung & $\mathbf{1 8 2 ( 6 5 \% )}$ & $53(63 \%)$ & $55(47 \%)$ & $285(59 \%)$ \\
\hline d-V2 in Distanzstellung & $98(35 \%)$ & $31(37 \%)$ & $61(53 \%)$ & $195(41 \%)$ \\
\hline RS in Kontaktstellung & $309(74 \%)$ & $135(78 \%)$ & $193(73 \%)$ & $637(75 \%)$ \\
\hline RS in Distanzstellung & $109(26 \%)$ & $37(22 \%)$ & $70(27 \%)$ & $216(25 \%)$ \\
\hline
\end{tabular}

In den frühneuhochdeutschen Texten ist gelegentlich die Ausklammerung einer Satzkonstituente zu beobachten. Dadurch kann der Relativsatz im Nachfeld direkt an seinen Bezugsausdruck angeschlossen werden. Das gleiche kommt auch bei d-V2-Sätzen vor, deren einleitendes Pronomen so ebenfalls in Kontaktstellung zum ausgeklammerten Antezedens gelangt: 
darnach will ich fürbaß sagen von Palantina der schoenen junckfrawen / die [...] auff dem berg in Arrogon gelegen inbeschlossen was. (Melusine, S. 165)

(danach will ich weiter über die schöne Jungfrau Palantina erzählen, die [...] auf dem Berg in Aragon eingeschlossen war.)

vnd morgen wil ich zu üch bringen ainen frommen man / der můß sein tochter mit ym hye her zu eüch bringen. (Fortunatus, S. 451)

(und morgen will ich einen frommen Mann zu euch bringen, der muss seine Tochter mit ihm hierher zu euch bringen.)

Solche ausgeklammerten Antezedentien sind in den neueren Texten des Korpus erheblich seltener. Sie finden sich fast ausschließlich bei Präpositionalgliedern:

Ein Erbstück, fügte er hinzu. Von seinem Großvater, der übrigens auch Arzt gewesen sei. (Heißer Sommer, S. 168)

Ausklammerung des Antezedens in d-V2-Sätzen und Relativsätzen

\begin{tabular}{|l|l|l|l|l|}
\hline & $\begin{array}{l}\text { Frühneu- } \\
\text { hochdeutsch }\end{array}$ & $\begin{array}{l}\text { 19. Jahr- } \\
\text { hundert }\end{array}$ & $\begin{array}{l}\text { Gegenwarts- } \\
\text { sprache }\end{array}$ & Summe \\
\hline $\begin{array}{l}\text { d-V2 mit ausgeklam- } \\
\text { mertem Antezedens }\end{array}$ & 20 & 1 & 1 & 22 \\
\hline $\begin{array}{l}\text { RS mit ausgeklammertem } \\
\text { Antezedens }\end{array}$ & 31 & 4 & 4 & 39 \\
\hline
\end{tabular}

Betrachten wir nun die Distribution von d-V2-Sätzen und Relativsätzen in Abhängigkeit von der Klasse ihres Bezugsausdrucks. Wenn der Bezugsausdruck ein Pronomen ist, werden Relativsätze in allen drei untersuchten Perioden bevorzugt (Beispiel (35)). d-V2Sätze haben in der Mehrzahl volle Nominalphrasen als Antezedens (Beispiel (36)):

(35) Da gibt es halt die andern die es tatsächlich vermissen + dass nicht jemand da ist der ihnen ständig sacht was sie zu tun und zu lassen haben. (Bredel/Dittmar: ALEX B $51 \mathrm{~W}$ )

(36) Die Alte aber war eine böse Hexe, die lauerte den Kindern auf, und hatte bloß um sie zu locken ihr Brothäuslein gebaut. (KHM 15, S. 90)

Ausdrucksklasse des Antezedens von d-V2-Sätzen und Relativsätzen

\begin{tabular}{|l|l|l|l|l|}
\hline & $\begin{array}{l}\text { Frühneu- } \\
\text { hochdeutsch }\end{array}$ & $\begin{array}{l}\text { 19. Jahr- } \\
\text { hundert }\end{array}$ & $\begin{array}{l}\text { Gegenwarts- } \\
\text { sprache }\end{array}$ & Summe \\
\hline $\begin{array}{l}\text { d-V2 mit einem Pronomen } \\
\text { als Antezedens }\end{array}$ & $13(5 \%)$ & $2(2,5 \%)$ & $3(2,5 \%)$ & $18(4 \%)$ \\
\hline $\begin{array}{l}\text { d-V2 mit voller NP als An- } \\
\text { tezedens }\end{array}$ & $201(72 \%)$ & $80(95 \%)$ & $90(77,5 \%)$ & $371(77 \%)$ \\
\hline $\begin{array}{l}\text { d-V2 mit einem Satz als An- } \\
\text { tezedens }\end{array}$ & $66(23 \%)$ & $2(2,5 \%)$ & $23(20 \%)$ & $91(19 \%)$ \\
\hline $\begin{array}{l}\text { RS mit einem Pronomen als } \\
\text { Antezedens }\end{array}$ & $87(21 \%)$ & $13(8 \%)$ & $37(14 \%)$ & $137(16 \%)$ \\
\hline $\begin{array}{l}\text { RS mit voller NP als Ante- } \\
\text { zedens }\end{array}$ & $310(74 \%)$ & $159(92 \%)$ & $226(86 \%)$ & $695(81 \%)$ \\
\hline $\begin{array}{l}\text { RS mit einem Satz als Ante- } \\
\text { zedens }\end{array}$ & $21(5 \%)$ & 0 & 0 & $21(3 \%)$ \\
\hline \multicolumn{1}{|c|}{ Summe } & $418(100 \%)$ & $172(100 \%)$ & $263(100 \%)$ & $853(100 \%)$ \\
\hline
\end{tabular}


Wie man sieht, sind die große Mehrzahl der Antezedentien sowohl von d-V2-Sätzen als auch von Relativsätzen volle Nominalphrasen. Es kommen aber auch etliche Beispiele vor, in denen sich ein d-V2-Satz auf den gesamten vorausgehenden Satz bezieht:

(38) sÿ sahen das Reÿmund die hirß haut so schmal vnd klein [...] geschnitten haet / das nam sÿ alle gar ser wunder. (Melusine, S. 23)

(Sie sahen, dass Reymund die Hirschhaut so schmal und kleingeschnitten hatte, das wunderte sie alle sehr.)

(39) Er hatte sie absichtlich verletzt, das wurde ihm plötzlich quälend bewusst. (Heißer Sommer, S. 12)

Wird das d-Pronomen von einer Präposition regiert, so sind Relativsätze in allen untersuchten Perioden häufiger als d-V2-Sätze:

(40) da funde er etwann ain alte witwen / mit der er sich reyssen woldt. (Fortunatus, S. 409) (Da fand er irgendwann eine alte Witwe, die er an sich reißen wollte.)

(41) Das wird wohl eine Kunst sein, von der ich auch nichts verstehe. (KHM 4, S. 38)

(42) Aber Ullrich hatte gezögert und schließlich auf den Tisch gezeigt, an dem die beiden saßen. (Heißer Sommer, S. 13)

Anzahl der durch eine Präposition regierten d-Pronomen an den d-V2-Sätzen und Relativsätzen im Korpus

\begin{tabular}{|l|l|l|l|l|l|}
\hline & $\begin{array}{l}\text { Frühneu- } \\
\text { hochdeutsch }\end{array}$ & $\begin{array}{l}\text { 19. Jahr- } \\
\text { hundert }\end{array}$ & $\begin{array}{l}\text { Gegen- } \\
\text { warts- } \\
\text { sprache }\end{array}$ & Summe & $\begin{array}{l}\text { Summe d-Pronomen } \\
\text { ohne Präp.-Rektion }\end{array}$ \\
\hline $\begin{array}{l}\text { d-V2 mit } \\
\text { Präp.+d- } \\
\text { Pronomen }\end{array}$ & 3 & 3 & 3 & $9(2 \%)$ & $471(98 \%)$ \\
\hline $\begin{array}{l}\text { RS mit Präp.+d- } \\
\text { Pronomen }\end{array}$ & 33 & 14 & 48 & $95(11 \%)$ & $758(89 \%)$ \\
\hline
\end{tabular}

Schließlich ist darauf hinzuweisen, dass ein d-V2-Satz einen Bezugsausdruck haben kann, in dem ein Relativsatz enthalten ist (vgl. den fettgedruckten Bezugsausdruck in Beispiel (44)). Der umgekehrte Fall ist dagegen nicht möglich. Der Relativsatz in (44a) schließt an eine Satzkonstituente an, die einen d-V2-Satz enthält. Ein solcher Anschluss ist ungrammatisch:

(44) Heute Nacht kommen zwölf schwarze Männer, die mit Ketten behangen sind, die werden dich fragen. (KHM 4, S. 40)

(44a) *Heute Nacht kommen zwölf schwarze Männer, die sind mit Ketten behangen, die dich fragen werden.

Zuletzt untersuche ich nun die semantischen Beziehungen von Relativsätzen und d-V2Sätzen zu ihren Antezedentien. Obgleich d-V2-Sätze in seltenen Fällen restriktive Funktion haben können (vgl. Faucher 1984, S. 21: Es gibt welche unter uns, die machen nicht mehr mit, Huard 2006, S. 119: Es gibt Dinge, die kann man nicht kaufen und Gärtner 2000, 2001), treten sie in meinem Korpus ausschließlich in appositiver Verwendung auf, wie etwa in Beispiel (45). Nur Relativsätze kommen in meinen Daten restriktiv vor (Beispiel (46)): 
(45) Und die saßen da drinne diese zöllner und und grenzsoldaten die habn selbst ne janz blöde figur abgegeben. (Bredel/Dittmar: B 130 )

(46) Derjenige, der gerade redet, fragt, ob das Oberseminar von Renke gesprengt werden soll. (Heißer Sommer, S. 123)

(47) d-V2-Sätze und Relativsätze mit restriktiver und appositiver Funktion im untersuchten Korpus

\begin{tabular}{|c|c|c|c|c|}
\hline & $\begin{array}{l}\text { Frühneu- } \\
\text { hochdeutsch }\end{array}$ & $\begin{array}{l}\text { 19. Jahr- } \\
\text { hundert }\end{array}$ & $\begin{array}{l}\text { Gegenwarts- } \\
\text { sprache }\end{array}$ & Summe \\
\hline d-V2 restriktiv & $3(1 \%)$ & $0(0 \%)$ & $1(1 \%)$ & $4(1 \%)$ \\
\hline d-V2 appositiv & $277(99 \%)$ & $84(100 \%)$ & $115(99 \%)$ & $476(99 \%)$ \\
\hline Summe & 280 & 84 & 116 & 480 \\
\hline RS restriktiv & $99(24 \%)$ & $25(15 \%)$ & $61(23 \%)$ & $173(20 \%)$ \\
\hline RS appositiv & $319(76 \%)$ & $147(85 \%)$ & $202(77 \%)$ & $668(78 \%)$ \\
\hline Summe & 418 & 172 & 263 & 853 \\
\hline
\end{tabular}

Wie man sieht, sind bis auf sehr wenige Ausnahmen alle d-V2-Sätze im Korpus nichtrestriktiv. Unter den Relativsätzen sind in meinem Korpus deutlich mehr appositive als restriktive. Das mag überraschend erscheinen, da restriktive Relativsätze im Normalfall weitaus häufiger vorkommen als appositive (vgl. Lehmann 1984, S. 279). In meinem Korpus könnte die hohe Frequenz appositiver Relativsätze darauf zurückzuführen sein, dass es sich um erzählende Texte handelt. Die Relativsätze dienen in vielen Fällen nicht der Identifizierung der Referenten, sondern bringen die Handlung voran, indem sie neue Sachverhalte und Ereignisse einführen bzw. zusätzliche Einzelheiten der Figuren und Gegenstände beschreiben.

\section{Abschließende Bemerkungen}

Meine Untersuchung zeigt, dass d-V2-Sätze in allen drei Perioden des Korpus vorkommen. Obwohl die frühneuhochdeutsche Syntax von der gegenwartsdeutschen in Vielem verschieden ist, hat sich herausgestellt, dass d-V2-Sätze als Alternativformen zu Relativsätzen schon im Frühneuhochdeutschen auftreten.

Wie die Korpusuntersuchung deutlich macht, verhalten sich Relativsätze insgesamt flexibler als d-V2-Sätze. Man vergleiche hierzu die Tabellen (25), (43) und (47). Anders als d-V2-Sätze können Relativsätze im Vor- und Mittelfeld stehen; viel häufiger als dV2-Sätze sind sie semantisch restriktiv; viel häufiger als die d-Pronomina in d-V2Sätzen treten Relativpronomina unter Rektion von Präpositionen auf; bei pronominalen Antezedentien werden Relativsätze gegenüber d-V2-Sätzen bevorzugt. Diese Tendenzen zeigen sich gleichermaßen in den drei untersuchten sprachgeschichtlichen Perioden.

Eigenschaften, die demnach für d-V2-Sätze charakteristisch sind, sind die Stellung nach der rechten Satzklammer und Appositivität. Unter folgenden Bedingungen können Relativsätze gut durch d-V2-Sätze ersetzt werden: 
(a) bei Nachstellung,

(b) in Distanz-oder Kontaktstellung zum Antezedens,

(c) wenn das Antezedens eine volle Nominalphrase oder ein Satz ist,

(d) wenn das d-Pronomen nicht durch eine Präposition regiert ist,

(e) bei Appositivität.

In diesem Zusammenhang sind die Grimmschen Märchen nochmals erwähnenswert. Die Brüder Grimm haben bei der Bearbeitung ihrer Texte aus stilistischen Gründen viele Relativsätze durch d-V2-Sätze ersetzt. Dies war jedoch nur unter den herausgearbeiteten Bedingungen möglich. Bei Vor- oder Mittelfeldstellung, pronominalen Antezedentien, Vorhandensein einer regierenden Präposition und bei Restriktivität wurden die Relativsätze beibehalten.

Zur Illustration noch je ein Beispiel aus den drei untersuchten Perioden, die den Gebrauch von d-V2-Sätzen nach den oben aufgezählten Bedingungen veranschaulichen. Alle drei weisen Stellung nach der rechten Satzklammer, Kontaktstellung zum Antezedens, volle Nominalphrasen als Antezedentien, keine regierende Präposition und appositiven Gebrauch auf:

(48) da legte man die schoen junckfrawen Herminam nider / vnd auch zů ir den Vriens iren gemahel / die lebten so lieblich die selb nacht mit einander. (Melusine, S. 60)

(Da legte man die schöne Jungfrau Hermina nieder, und neben ihr den Vriens, ihren Gemahl, die verlebten so lieblich die Nacht miteinander.)

(49) Da trat ein Mann herein, der war größer als alle andere. (KHM 4, S. 41)

(50) Dafür bekommt man einen Schein, den braucht man fürs Examen. (Heißer Sommer, $\mathrm{S}$. 28)

Meine Untersuchung führt zu der Schlussfolgerung, dass d-V2-Sätze nicht als Relativsätze interpretiert werden können. Das Einleitungspronomen der/die/das hat in d-V2Sätzen demonstrative Funktion und fungiert nicht als Relativum.

\section{Literatur}

\section{Quellen}

Bredel, Ursula/Dittmar, Norbert (1999): Transkripte und Hörbeispiele aus dem Ost-West-Korpus „Kollektives Gedächtnis“. In: http://userpage.fu-berlin.de/ nordit/HP/

Der Spiegel (1993): COSMAS II - Institut für Deutsche Sprache, Mannheim.

Fortunatus. In: J. D. Müller (Hg.): Romane des 15. und 16. Jahrhunderts. Nach den Erstdrucken mit sämtlichen Holzschnitten. Frankfurt am Main: Deutscher Klassiker Verlag. S. 383-580.

Grimm, Jacob und Wilhelm (1985): Kinder- und Hausmärchen. Gesammelt durch die Brüder Grimm. Vollständige Ausgabe auf der Grundlage der dritten Auflage (1837), hrsg. von H. Rölleke. Frankfurt am Main: Deutscher Klassiker Verlag.

Grimm, Jacob und Wilhelm (1989): Kinder- und Hausmärchen. Ausgabe letzter Hand mit den Originalanmerkungen der Brüder Grimm. Mit einem Anhang sämtlicher, nicht in allen Auflagen veröffentlichten Märchen und Herkunftsnachweisen. 3 Bde., hrsg. von H. Rölleke. Stuttgart: Reclam. 
von Ringoltingen, Thüring (1990): Melusine. In: J. D. Müller (Hg.), Romane des 15. und 16. Jahrhunderts. Nach den Erstdrucken mit sämtlichen Holzschnitten. Frankfurt am Main: Deutscher Klassiker Verlag. S. 9-176.

Timm, Uwe (1985): Heißer Sommer. Köln: Kiepenheuer \& Witsch.

\section{Wissenschaftliche Literatur}

Bergmann, Rolf (1985): Relativsatz-Probleme in Grammatiken der deutschen Gegenwartssprache. In: Koller, E./Moser, H. (Hg.): Studien zur deutschen Grammatik. Johannes Erben zum 60. Geburtstag. Innsbruck: Innsbrucker Beiträge zur Kulturwissenschaft. S. 51-66.

Faucher, Eugène (1984): L'ordre pour la clôture. Essai sur la place du verbe en allemand. Nancy: P.U.N.

Gärtner, Hans-Martin (2000): Are there V2 relative clauses in German? In: The Journal of Comparative Germanic Linguistics 3, S. 97-141.

Gärtner, Hans-Martin (2001): Bound Focus and Assertionality from V2-Relatives. http://www.zas.gwzberlin.de/mitarb/homepage/gaertner/files/.

Holler, Anke (2005): Weiterführende Relativsätze. Empirische und theoretische Aspekte. Berlin: Akademie Verlag.

Huard, René (2006): Il n'y a pas de relative à verbe second (I). In: Nouveaux cahiers d'allemand 2, S. 111 128.

Lehmann, Christian (1995): Relativsätze. In: Jacobs, J./von Stechow, A. (Hg.): Syntax. Ein internationales Handbuch zeitgenössischer Forschung, Berlin/New York: de Gruyter. S. 1199-1216.

Penner, Zvi (1996): From Empty to Doubly-Filled Complementizers. A Case Study in the Acquisition of Subordination in Bernese Swiss German. Fachgruppe Sprachwissenschaft der Universität Konstanz.

Ravetto, Miriam (2006): Es war einmal ein Königssohn, der bekam Lust in der Welt umher zu ziehen. Le 'false relative' in tedesco. Vercelli: Mercurio.

Reis, Marga (1997): Zum syntaktischen Status unselbständiger Verbzweit-Sätze. In: Dürscheid, C./Ramers, K.H./Schwarz, M. (Hg.): Sprache im Fokus. Festschrift für Heinz Vater zum 65. Geburtstag. Tübingen: Niemeyer. S. 121-144.

Schieb, Gabriele (1978): Der Verbkomplex aus verbalen Bestandteilen. In: Kettmann, G./Schildt, J.: Zur Ausbildung der Norm der deutschen Literatursprache auf der syntaktischen Ebene (1470 - 1730). Der Einfachsatz. Berlin: Akademie-Verlag. S. 39-234.

Wiltschko, Martina (1998): On the Syntax and Semantics of (Relative) Pronouns and Determiners. In: Journal of Comparative Germanic Linguistics 2, S. 143-181.

Wöllstein-Leisten, Angelika/Heilmann, Axel/Stepan, Peter/Vikner, Sten (1997): Deutsche Satzstruktur. Grundlagen der syntaktischen Analyse. Tübingen: Stauffenburg.

Zifonun, Gisela (2001): Grammatik des Deutschen im europäischen Vergleich: der Relativsatz. Mannheim: Institut für Deutsche Sprache. Amades 3/01. (Arbeitspapiere und Materialen zur deutschen Sprache).

\section{Dr. Miriam Ravetto}

Università degli Studi del Piemonte Orientale Amadeo Avogadro

Facoltà di lettere e filosofia

Dipartimento di Studi Umanistici

Via Alessandro Manzoni, 8

13100 Vercelli

Italien

miriamravetto@Lett.unipmn.it 